\title{
ALTERAÇÃO DA EFICIÊNCIA DO 1-MCP COM O RETARDO NA SUA APLICAÇÃO APÓS A COLHEITA EM MAÇÃSS 'FUJI SUPREMA'
}

\author{
CASSANDRO VIDAL TALAMINI DO AMARANTE², LUIZ CARLOS ARGENTA ${ }^{3}$, \\ MARCELO JOSÉ VIEIRA ${ }^{4}$, CRISTIANO ANDRÉ STEFFENS ${ }^{5}$
}

RESUMO - Estudos indicam que a eficiência do 1-metilciclopropeno (1-MCP) para conservação pós-colheita de maçãs é máxima quando aplicado até uma semana após a colheita. No entanto, o carregamento das câmaras de armazenagem comerciais com maçãs 'Fuji' pode extender-se por mais de uma semana. Os efeitos da aplicação tardia do 1-MCP para maçãs 'Fuji' não têm sido reportados. Este trabalho teve por objetivo avaliar os efeitos do retardo na aplicação de 1-MCP, a partir da data de colheita, na preservação da firmeza de polpa, acidez titulável (AT) e sólidos solúveis (SS), e na prevenção de escaldadura superficial, escurecimento da polpa e podridões em maçãs 'Fuji Suprema'. Os frutos foram colhidos em pomares comerciais do Estado de Santa Catarina, nas regiões de Fraiburgo (quatro pomares), Bom Retiro (três pomares) e São Joaquim (três pomares), em abril de 2006. Os frutos foram refrigerados $12 \mathrm{~h}$ após a colheita e mantidos durante nove meses sob atmosfera do ar a $0,5 \pm 0,5{ }^{\circ} \mathrm{C}$ e $90-95 \%$ de UR. Os frutos foram tratados com ar (controle) ou 1-MCP (1 $\mu \mathrm{L} . \mathrm{L}^{-1}$, durante 24h), na mesma temperatura de armazenamento, aos $0 ; 4 ; 8 ; 12 ; 16$ ou 20 dias após a colheita. A qualidade dos frutos foi determinada após o armazenamento refrigerado, mais sete dias a $23{ }^{\circ} \mathrm{C}$. O retardo na aplicação do $1-\mathrm{MCP}$, por até 20 dias após a colheita, não reduziu sua eficiência na conservação da firmeza da polpa e na prevenção do escurecimento da polpa e podridões, em frutos colhidos nas três regiões, bem como na preservação do teor de SS em frutos colhidos em Bom Retiro e São Joaquim. No entanto, a eficiência do 1-MCP sobre a prevenção da escaldadura superficial foi reduzida quando sua aplicação foi atrasada por 16 ou 20 dias a partir da data de colheita, especialmente nos frutos das regiões que apresentaram maior suscetibilidade ao distúrbio (Fraiburgo e São Joaquim). Os benefícios do 1-MCP sobre a conservação da AT foram reduzidos quando aplicado 20 dias após a colheita nos frutos da região Bom Retiro. Os resultados deste estudo evidenciam os benefícios do tratamento 1-MCP na conservação da qualidade de maçãs 'Fuji Suprema', armazenadas sob atmosfera do ar, por longos perídos, e a redução da eficiência deste tratamento na prevenção da escaldadura superficial, quando a aplicação for atrasada em mais de 12 dias após a colheita dos frutos.

Termos de indexação: Escaldadura superficial, escurecimento de polpa, Malus domestica, podridões, qualidade.

\section{CHANGES OF 1-MCP EFFICIENCY BY DELAYING ITS POSTHARVEST APPLICATION ON 'FUJI SUPREMA'APPLES}

\begin{abstract}
It has been shown that the maximum efficiency of 1-methylcyclopropene (1-MCP) for postharvest preservation of apples is achieved when fruit are treated up to one week after harvesting. However, the full loading of commercial stores with 'Fuji' apples might take more than one week. The effects of delays for 1-MCP treatment of 'Fuji' apples have not been reported. This work was carried out to assess the effects of delaying treatment with 1-methylcyclopropene (1-MCP), after fruit harvest, on flesh firmness, titratable acidity (TA); soluble solids content (SSC), as well as on prevention of superficial scald, core browning, and decay of 'Fuji Suprema' apples. Fruit were harvested in commercial orchards of the State of Santa Catarina, located at the regions of Fraiburgo (four orchards), Bom Retiro (three orchards), and São Joaquim (three orchards), in April of 2006. Fruits were cold stored $12 \mathrm{~h}$ after harvesting, and left during nine months in air at $0.5 \pm 0.5^{\circ} \mathrm{C}$ and $90-95 \% \mathrm{RH}$. Fruit were treated with air (control) or $1-\mathrm{MCP}\left(1 \mu \mathrm{L} . \mathrm{L}^{-1}\right.$, for $24 \mathrm{~h}$ ), in hermetical chambers, at harvest, or after $4,8,12,16$, or 20 days at $0.5^{\circ} \mathrm{C}$. Fruit quality was assessed after removal from cold storage, followed by seven days at $23{ }^{\circ} \mathrm{C}$. The delay of 1 -MCP treatment, for up to 20 days after fruit harvesting, did not reduce its efficiency to preserve flesh firmness and to prevent core browning and decay, in fruit from the three regions, as well as to preserve the SSC in fruits from Bom Retiro and São Joaquim. However, the efficiency of 1-MCP to prevent superficial scald was reduced when its application was delayed by 16 or 20 days after harvesting, especially in apples from regions with a high susceptibility to the disorder (Fraiburgo and São Joaquim). The benefits of 1-MCP to preserve the TA were reduced in fruit from Bom Retiro, treated 20 days after harvest. These results show the benefits of 1-MCP to preserve fruit quality of 'Fuji Suprema' during long term storage in air, and the loss of its efficiency to prevent superficial scald with delays of fruit treatment superior to 12 days after harvesting.
\end{abstract}

Index terms: Decay, flesh browning, Malus domestica, quality, superficial scald.

\footnotetext{
1(Trabalho 224-09). Recebido em: 29-09-2009. Aceito para publicação em: 27-05-2010.

${ }^{2}$ Ph.D., Bolsista de Produtividade em Pesquisa do CNPq. Prof. do Dept ${ }^{\circ}$. de Agronomia - CAV/UDESC Lages-SC.E-mail: amarante@cav.udesc.br ${ }^{3}$ Dr., Pesquisador da Epagri, Estação Experimental de Caçador. Caçador-SC. E-mail: argenta@epagri.sc.gov.br

${ }^{4}$ M.Sc., Epagri, Estação Experimental de Caçador. Caçador-SC. E-mail: marcjv@hotmail.com

${ }^{5}$ Dr., Prof. do Dept ${ }^{\circ}$. de Agronomia, CAV/UDESC. Lages-SC. E-mail: steffens@cav.udesc.br
} 


\section{INTRODUÇÃO}

O cultivo nacional de maçãs concentra-se na região Sul, com cerca de $40 \%$ da produção representada pela cultivar 'Fuji' e seus clones (ABPM, 2009). Maçãs deste grupo são suscetíveis ao dano por $\mathrm{CO}_{2}$ quando armazenadas em condição de atmosfera controlada (AC) (ARGENTA et al., 1994; 2002) e mais suscetíveis a podridões pós-colheita que outras cultivares (VALDEBENITO SANHUEZA et al., 2006). As perdas da produção de maçãs 'Fuji', causadas por podridões pós-colheita, podem chegar a $40 \%$, sendo tanto maiores quanto maior o retardo na colheita, devido ao maior período de ocorrência de infecções latentes nos frutos ainda no pomar (VALDEBENITO SANHUEZA, 1996).

Mais de $90 \%$ dos novos plantios de maçãs 'Fuji', no Sul do Brasil, têm sido com o clone 'Fuji Suprema', por apresentar uma coloração vermelha sólida, que cobre mais de $80 \%$ do fruto. O rápido desenvolvimento de cor vermelha na epiderme permite a colheita precoce dos frutos, o que reduz os riscos de dano por $\mathrm{CO}_{2}$ durante armazenamento em AC (ARGENTA et al., 2002), bem com a ocorrência de podridões pós-colheita (ARGENTA; MONDARDO, 1994). No entanto, a colheita precoce aumenta os riscos de escaldadura superficial, especialmente em maçãs 'Fuji', que apresentam maior suscetibilidade ao distúrbio que maçãs 'Gala' (EMONGOR et al., 1994). O risco de escaldadura superficial varia entre regiões, sendo menor em locais com clima mais úmido e frio, no período que antecede a colheita (EMONGOR et al., 1994). A causa provável da escaldadura superficial é a biossíntese de $\alpha$-farneseno no tecido da casca, e sua subsequente oxidação a trienos conjugados, ocasionando a degradação de células e o escurecimento do tecido (JU; CURRY, 2000). $\mathrm{O}$ etileno promove a biossíntese de $\alpha$-farneseno e, portanto, aumenta a incidência de escaldadura (EMONGOR et al., 1994).

O tratamento pós-colheita de maçãs com 1-metilciclopropeno (1-MCP) reduz a produção de etileno e a respiração (FAN et al., 1999a; SAFTNER et al., 2003; AKBUDAK et al., 2009), retarda a perda de firmeza de polpa, acidez titulável e sólidos solúveis (ARGENTA et al., 2001a; SAFTNER et al., 2003; ZANELLA, 2003; AKBUDAK et al., 2009), reduz a ocorrência de escaldadura superficial (FAN et al., 1999b; RUPASINGHE et al., 2000; DEELL et al., 2002; ZANELLA, 2003; ARGENTA et al., 2005; JUNG; WATKINS, 2008; AKBUDAK et al., 2009) e escurecimento interno da polpa (FAN et al., 1999b), e pode reduzir a incidência de podridões póscolheita (SAFTNER et al., 2003). Todavia, os efeitos do 1-MCP em maçãs dependem da cultivar e da condição de armazenamento (WATKINS et al., 2000; ARGENTA et al., 2001a; AKBUDAK et al., 2009), bem como da interação entre concentração do produto e estádio de maturação do fruto (ARGENTA et al., 2005; WATKINS; NOCK, 2005; JUNG; WATKINS, 2008). O tratamento de maçãs 'Fuji' com 1-MCP na colheita preserva a qualidade durante armazenagem de longo prazo (seis meses) a $0{ }^{\circ} \mathrm{C}$, em atmosfera do ar (FAN et al., 1999a). Todavia, menores benefícios do 1-MCP são observados em maçãs colhidas em estádio avançado de maturação (ARGENTA et al., 2005) ou tratadas depois de decorrido certo tempo de armazenamento refrigerado (ARGENTA et al., 2005; WATKINS; NOCK, 2005; JUNG;WATKINS, 2008). A menor eficiência do 1-MCP nestas condições é resultado do maior acúmulo de transcritos dos genes das rotas de biossíntese de etileno e de $\alpha$-farneseno (PECHOUS et al., 2005; TSANTILI et al., 2007).

Por outro lado, o tratamento de maçãs 'Fuji' com 1-MCP, imediatamente após a colheita, pode estender o período de maior suscetibilidade dos frutos ao dano por $\mathrm{CO}_{2}$, que ocorre no início do armazenamento refrigerado (ARGENTA et al., 2001b). Desta forma, o retardo no tratamento de maçãs 'Fuji' com 1-MCP, após a colheita, pode ser desejável como forma de reduzir o risco de dano por $\mathrm{CO}_{2}$, especialmente quando os frutos são armazenados sob atmosfera controlada.

Em maçãs 'Fuji Suprema', colhidas em estádio menos avançado de maturação, visando a reduzir principalmente podridões pós-colheita, o tratamento com 1-MCP pode reduzir a suscetibilidade à escaldadura superficial, além de retardar o amadurecimento. No entanto, em várias situações, o enchimento de câmaras comerciais de armazenagem pode demorar até 20 dias, levando ao retardo da aplicação do 1-MCP. Isto pode ocorrer em pequenas empresas (produtores), com baixo volume de produção a ser colhida, em várias passadas nos pomares, com o armazenamento em uma única câmara. $\mathrm{O}$ retardo no tratamento dos frutos com 1-MCP também pode ocorrer em grandes empresas, nas primeiras passadas de colheita, quando há baixo volume de frutos, e os mesmos são destinados à armazenagem em câmaras muito grandes, ou quando há classificação dos frutos destinados à exportação, antes do seu armazenamento e tratamento com 1-MCP.

O presente estudo teve por objetivo avaliar os efeitos do retardo no tratamento com 1-MCP a partir da data de colheita, na preservação dos atributos físico-químicos e na prevenção de escaldadura superficial, escurecimento interno e podridões, em maçãs 'Fuji Suprema', colhidas em três regiões de 
produção no Estado de Santa Catarina.

\section{MATERIAL E MÉTODOS}

Maçãs 'Fuji Suprema' foram colhidas em pomares comerciais nas regiões de Fraiburgo (situada a $27^{\circ} 01^{\prime}$ de latitude $\mathrm{S}$ e $50^{\circ} 55^{\prime}$ de longitude $\mathrm{W}$, em quatro pomares, com altitude entre 950-1.000 m), Bom Retiro (situada a $22^{\circ} 34^{\prime}$ de latitude $\mathrm{S}$ e $46^{\circ} 45^{\prime}$ de longitude $\mathrm{W}$, em três pomares, com altitude entre $1.000-1.100 \mathrm{~m}$ ) e São Joaquim (situada a $28^{\circ} 15^{\prime}$ de latitude $\mathrm{S}$ e $49^{\circ} 54^{\prime}$ 'de longitude $\mathrm{W}$, em três pomares, com altitude entre 1.200-1.300 m). Os frutos foram colhidos na $1^{\mathrm{a}}$ semana de abril em Fraiburgo, e na $2^{\mathrm{a}}$ semana de abril em São Joaquim e Bom Retiro, no ano de 2006.

Os frutos foram refrigerados $12 \mathrm{~h}$ após a colheita e mantidos durante nove meses, a $0,5 \pm$ $0,5{ }^{\circ} \mathrm{C} / 90-95 \%$ de UR, sob atmosfera do ar. Os frutos foram submetidos ao tratamento com ar (CK, controle) ou 1-MCP $\left(1 \mu \mathrm{L} . \mathrm{L}^{-1}\right)$, na mesma temperatura de armazenamento, $0 ; 4 ; 8 ; 12 ; 16$ ou 20 dias após a colheita.

Os frutos receberam tratamento com 1-MCP em câmaras herméticas de aproximadamente $0,340 \mathrm{~m}^{3}$, durante 24 horas. A dose $\left(1 \mu \mathrm{L} . \mathrm{L}^{-1}\right)$ e o tempo de exposição ao 1-MCP (24 horas) corresponderam àquelas indicadas como mais eficientes para maçãs (ARGENTA et al., 2005). Durante o período de tratamento com 1-MCP, os frutos-controle (CK) foram mantidos em câmara hermética igual à usada para tratamento com 1-MCP, sob a mesma temperatura. O gás de 1-MCP foi gerado misturando-se SmartFresh ${ }^{\mathrm{SM}}$ (AgroFresh Inc., Philadelphia-PA, EUA) e água a $35^{\circ} \mathrm{C}$, num frasco $\mathrm{de} \approx 0,5 \mathrm{~L}$, e bombeado à câmara de tratamento em sistema fechado, por um período necessário para alcançar a concentração desejada. A concentração de 1-MCP no ar da câmara de tratamento foi determinada por meio de um cromatógrafo a gás (Shimadzu 14B, Tóquio) equipado com um detector de ionização de chama e coluna de vidro de $2,5 \mathrm{~m}$ e diâmetro interno de 3,2 mm, empacotada com Poropak Q, 80 a 100 mesh, usando 1-MCP como padrão. As temperaturas do forno, do detector e do injetor, foram de 90; 200 e $100{ }^{\circ} \mathrm{C}$, respectivamente. Os fluxos de $\mathrm{N}_{2}, \mathrm{H}_{2}$ e ar utilizados foram de $25 ; 25$ e $300 \mathrm{~mL} \mathrm{~min}^{-1}$, respectivamente.

$\mathrm{Na}$ colheita, os frutos foram analisados quanto à firmeza da polpa, acidez titulável (AT), sólidos solúveis (SS) e índice de iodo-amido. Após nove meses de armazenamento refrigerado, mais sete dias a $23{ }^{\circ} \mathrm{C}$, os frutos foram analisados quanto à firmeza da polpa, AT, SS e incidência de escaldadura superficial, escurecimento de polpa e podridões.

A firmeza da polpa foi medida em dois lados opostos da superfície de cada fruto, após a remoção da casca, pela utilização de um penetrômetro com ponteira de $11 \mathrm{~mm}$ (Güss, África do Sul). Os valores de SS e AT foram determinados através de suco preparado com espremedor tipo Champion (Plastaket Mgf, CA). O teor de SS (\%) foi medido usando-se refratômetro digital com compensação automática de temperatura (Atago, Japão). A AT (\% de ácido málico) foi determinada utilizando-se de $5 \mathrm{~mL}$ do suco do fruto, diluído em $20 \mathrm{~mL}$ de água destilada, sendo esta solução titulada com $\mathrm{NaOH} 0,1 \mathrm{~N}$ até $\mathrm{pH}$ 8,1 , usando um titulador automático (Radiometer, Copenhagen, Dinamarca). O índice de iodo-amido foi avaliado numa escala de 1 (secção transversal da polpa corada com iodo, indicando alto teor de amido e fruto imaturo) a 9 (secção transversal da polpa não corada com iodo, indicando baixo teor de amido e fruto maduro).

A incidência (\%) de escaldadura superficial, escurecimento interno e podridões foi avaliada pela contagem dos frutos com sintoma em relação ao número total de frutos. A avaliação de escurecimento interno difuso de polpa foi feita na secção transversal dos tecidos da região carpelar, em frutos cortados na região equatorial.

Foi utilizada uma caixa contendo 85 frutos, em cada combinação de região de produção, pomar, tratamento (CK e 1-MCP) e data de aplicação do 1-MCP. Os dados de cada região foram analisados separadamente. Foi realizada análise de variância (ANOVA) e teste de comparação de médias (LSD; $\mathrm{p}<0,05)$, visando a avaliar os efeitos do tratamento com 1-MCP e do retardo na sua aplicação após a colheita, sobre os diferentes atributos de qualidade avaliados, utilizando o programa SAS (SAS Institute, 2002). Dados em porcentagem foram transformados através da fórmula arco-seno $[(\mathrm{x}+0,5) / 100]^{1 / 2}$ antes de serem submetidos à ANOVA.

\section{RESULTADOS E DISCUSSÃO}

Os frutos das três regiões apresentaram firmeza de polpa $>70 \mathrm{~N}$ e índice de amido $<6$ no dia da colheita (Tabela 1), indicando que eles estavam em estádio de maturação adequado para armazenamento refrigerado de médio a longo prazos (ARGENTA e al., 2001a; 2005). Os frutos colhidos em pomares da região de Bom Retiro encontravamse em estádio mais avançado de maturação (menor firmeza de polpa e maior índice de iodo-amido) do que aqueles colhidos nas regiões de Fraiburgo e São Joaquim, sendo que, neste último, os frutos 
apresentavam valores maiores de firmeza de polpa (Tabela 1).

Frutos tratados com 1-MCP apresentaram maior firmeza da polpa em relação aos frutos-controle, nas três regiões de produção, independentemente do atraso de sua aplicação após a colheita (Tabela 2). Considerando os valores médios nas três regiões, frutos tratados com 1-MCP apresentaram firmeza de polpa superior $(\sim 30 \%)$ em relação aos frutos do tratamento-controle (correspondente a 21; 20 e 26 $\mathrm{N}$, nos frutos colhidos em Bom Retiro, Fraiburgo e São Joaquim, respectivamente), após nove meses de armazenamento refrigerado, seguido de uma semana à temperatura ambiente (Tabela 2). $\mathrm{O}$ valor mínimo de firmeza de polpa para venda ao mercado consumidor, normalmente adotado pelas empresas que comercializam maçãs 'Fuji' no Sul do Brasil, é de $53 \mathrm{~N}$ (12 libras), não podendo ser inferior a 44,5 N (10 libras) (BRASIL, 2006). Ao final de nove meses de armazenamento refrigerado, seguido de uma semana à temperatura ambiente, frutos do tratamento-controle apresentaram firmeza de polpa próxima destes valores (49-53 N), enquanto frutos tratados com 1-MCP apresentaram valores de firmeza de polpa muito superiores $(71-78 \mathrm{~N})$ (Tabela 2).

A eficiência do tratamento 1-MCP para a manutenção da firmeza de polpa dos frutos não foi significativamente alterada pelo retardo da sua aplicação após a colheita, independentemente da região de produção. Esse resultado difere daqueles de Argenta et al. (2005) e Watkins e Nock (2005), os quais observaram significativa redução da eficiência do 1-MCP para a conservação da firmeza da polpa de maçãs 'Granny Smith' 'Law Rome' e 'Cortland' quando sua aplicação foi retardada por 14 a 21 dias após a colheita.

Nas três regiões, considerando a média dos diferentes períodos de retardo na aplicação do 1-MCP, frutos tratados com o produto apresentaram maiores valores de AT em relação aos frutoscontrole, com incrementos de 33; 36 e $45 \%$, nas regiões de São Joaquim, Fraiburgo e Bom Retiro, respectivamente (Tabela 2). Apenas em frutos colhidos na região de Bom Retiro, a AT reduziu com o retardo no tratamento dos frutos com 1-MCP, especialmente após 16 dias de armazenamento refrigerado. Da mesma forma, Argenta et al. (2005) não observaram redução significativa da eficiência do 1-MCP sobre a conservação da AT em maçãs 'Granny Smith' quando sua aplicação foi retardada em 15 ou 30 dias depois da colheita.

Em Bom Retiro e São Joaquim, considerando a média dos diferentes períodos de retardo na aplicação de 1-MCP, frutos tratados com o produto apresentaram maiores valores de SS em relação aos frutos-controle (Tabela 2). Em frutos destas duas regiões, não houve redução significativa no teor de SS com o retardo na aplicação do 1-MCP. Na região de Fraiburgo, não houve efeito do tratamento dos frutos com 1-MCP na preservação do teor de SS.

$\mathrm{O}$ tratamento com 1-MCP reduziu a incidência de escaldadura superficial, escurecimento de polpa e podridões em frutos colhidos nas três regiões, não sendo verificado aumento na incidência de escurecimento de polpa e de podridões com o retardo de até 20 dias no tratamento com o produto (Tabela 3). Frutos colhidos em Bom Retiro não apresentaram incidência de escaldadura superficial quando tratados com 1-MCP, mesmo com retardo de até 20 dias na aplicação do produto, enquanto frutos-controle apresentaram incidência do distúrbio pouco superior a $25 \%$. Frutos colhidos nas regiões de Fraiburgo e São Joaquim apresentaram maior suscetibilidade à escaldadura superficial em relação a Bom Retiro, apresentando incidência média de 82,4 e $74,5 \%$, respectivamente, quando não tratados com 1-MCP. A menor suscetibilidade dos frutos da região de Bom Retiro à escaldadura superficial deve-se, possivelmente, ao estádio mais avançado de maturação na colheita, em relação a frutos colhidos em Fraiburgo e São Joaquim (Tabela 1). Quanto mais avançado o estádio de maturação dos frutos na colheita, menor a suscetibilidade à escaldadura superficial (EMONGOR et al., 1994). Em frutos das regiões de Fraiburgo e São Joaquim, o tratamento com 1-MCP reduziu substancialmente a incidência de escaldadura superficial, quando feito até os 12 dias de armazenamento refrigerado. Em maçãs destas duas regiões, mesmo com retardo de 20 dias no tratamento com 1-MCP, a incidência de escaldadura foi cerca de $60-70 \%$ menor, comparativamente aos frutos não tratados.

Em maçãs 'Law Rome' e 'Cortland', que apresentam elevada suscetibilidade à escaldadura superficial, Jung e Watkins (2008) observaram que o retardo em sete dias após a colheita, para o tratamento dos frutos com 1-MCP ( $\left.1 \mu \mathrm{L} . \mathrm{L}^{-1}\right)$, no ambiente de armazenamento, reduziu substancialmente sua eficiência no controle do distúrbio, avaliado após seis meses de estocagem $\left(\right.$ a $\left.0,5^{\circ} \mathrm{C}\right)$ em atmosfera do ar, mais sete dias a $20^{\circ} \mathrm{C}$. Estes autores observaram incidência de escaldadura $>50 \%$, em frutos tratados com 1-MCP aos sete dias, e próximo de $100 \%$, em frutos tratados aos 21 dias de armazenamento refrigerado. Portanto, o controle de escaldadura superficial em maçãs com o 1-MCP depende da cultivar e do tempo decorrido entre a colheita e o 
tratamento com o produto (ARGENTA et al., 2005; WATKINS; NOCK, 2005; JUNG; WATKINS, 2008). Em maçãs 'Fuji Suprema' cultivadas no Sul do Brasil, o tratamento com 1-MCP, até 12 dias após o início do armazenamento refrigerado, reduziu substancialmente a sua incidência, em frutos provenientes de regiões com maior risco de manifestar o distúrbio (Tabela 3).

A suscetibilidade à incidência de escurecimento da polpa foi maior em frutos colhidos em São Joaquim, sendo moderada em Fraiburgo e baixa em Bom Retiro (Tabela 3). Todavia, nas três regiões, o tratamento com 1-MCP, até 20 dias após o início do armazenamento, reduziu a incidência deste distúrbio em mais de $80 \%$, comparativamente ao tratamentocontrole. Fan et al. (1999b) também reportaram menor severidade pós-colheita de escurecimento difuso de tecidos da região carpelar da polpa, considerado um dano relacionado à senescência dos frutos, em maçãs 'Fuji' e 'Granny Smith' tratadas com 1-MCP, como resultado da inibição na produção de etileno.

Frutos colhidos em Bom Retiro, Fraiburgo e São Joaquim, quando tratados com 1-MCP até 20 dias após o início do armazenamento, apresentaram reduções na incidência de doenças de $75 ; 86$ e $88 \%$, respetivamente, comparativamente aos frutos-controle (Tabela 3). Aparentemente, muitas das podridões observadas neste estudo desenvolveram-se a partir dos danos causados pela escaldadura superficial. Todavia, o retardo no amadurecimento, ocasionado pelo tratamento com 1-MCP, pode conferir aos tecidos dos frutos maior proteção à infecção de patógenos, reduzindo assim a incidência pós-colheita de doenças (SAFTNER et al., 2003).
Os resultados obtidos no presente estudo confirmam aqueles de trabalhos de outros autores, mostrando preservação da firmeza de polpa, da AT e do teor de SS (SAFTNER et al., 2003; ZANELLA, 2003), e redução na incidência de escaldadura superficial (FAN et al., 1999b; RUPASINGHE et al., 2000; DEELL et al., 2002; ZANELLA, 2003), escurecimento de polpa (FAN et al., 1999b) e doenças pós-colheita (SAFTNER et al., 2003) em maçãs, em resposta à aplicação do 1-MCP, devido ao seu efeito inibidor da ação do etileno (FAN et al., 1999a; SAFTNER et al., 2003; JUNG; WATKINS, 2008). Todavia, tem sido relatado redução na eficiência do tratamento com 1-MCP em retardar o amadurecimento e reduzir a incidência de distúrbios fisiológicos, em maçãs colhidas em estádio avançado de maturação (ARGENTA et al., 2005), ou tratadas após decorrido certo tempo de armazenamento refrigerado (ARGENTA et al., 2005; WATKINS; NOCK, 2005; JUNG; WATKINS, 2008). Neste trabalho, a redução na eficiência do 1-MCP, com o retardo na sua aplicação, foi verificada para o controle de escaldadura, somente em maçãs 'Fuji Suprema' colhidas em regiões com maior suscetibilidade ao distúrbio. Portanto, diferenças quanto à cultivar, região de produção e condições de nutrição e manejo das plantas no pomar, podem explicar as diferenças observadas por diversos autores, na resposta ao tratamento de maçãs com 1-MCP, em função do estádio fisiológico dos frutos. Nas condições de realização deste trabalho, foram observados benefícios significativos do tratamento de maçãs 'Fuji Suprema' com 1-MCP, na preservação da qualidade dos frutos, mesmo com um retardo de até 20 dias na sua aplicação após a colheita.

TABELA 1 - Atributos de maturação de maçãs 'Fuji Suprema' utilizadas no presente trabalho, colhidas em diferentes regiões de produção, em abril de 2006.

\begin{tabular}{lcccc}
\hline Região & $\begin{array}{c}\text { Firmeza de polpa } \\
(\mathrm{N})\end{array}$ & Índice de iodo-amido (1-9) & $\begin{array}{c}\text { Acidez titulável } \\
(\% \text { de ácido málico) }\end{array}$ & Sólidos solúveis (\%) \\
\hline Bom Retiro & $73,4 \mathrm{c}$ & $5,5 \mathrm{a}$ & $0,362 \mathrm{a}$ & $13,9 \mathrm{a}$ \\
Fraiburgo & $76,6 \mathrm{~b}$ & $4,4 \mathrm{~b}$ & $0,382 \mathrm{a}$ & $14,0 \mathrm{a}$ \\
São Joaquim & $83,3 \mathrm{a}$ & $4,2 \mathrm{~b}$ & $0,393 \mathrm{a}$ & $13,6 \mathrm{a}$ \\
\hline
\end{tabular}

Médias seguidas da mesma letra, nas colunas, não diferem entre si, pelo teste de $\operatorname{LSD}(\mathrm{p}<0,05)$. 
TABELA 2 - Atributos de firmeza de polpa, acidez titulável e sólidos solúveis de maçãs 'Fuji Suprema', colhidas em diferentes regiões de produção e tratadas com 1-MCP, após diferentes períodos de armazenamento refrigerado $\left(0,5 \pm 0,5^{\circ} \mathrm{C} / 90-95 \% \mathrm{UR}\right)$, sob atmosfera do ar, comparativamente ao tratamento-controle. Os frutos foram avaliados após nove meses de armazenamento refrigerado, mais sete dias a $23^{\circ} \mathrm{C}$.

\begin{tabular}{|c|c|c|c|c|c|c|}
\hline \multirow{2}{*}{$\begin{array}{l}\text { Aplicação do 1-MCP } \\
\text { (dias após a colheita) }\end{array}$} & \multicolumn{2}{|c|}{ Bom Retiro } & \multicolumn{2}{|c|}{ Fraiburgo } & \multicolumn{2}{|c|}{ São Joaquim } \\
\hline & Controle & 1-MCP & Controle & 1-MCP & Controle & 1-MCP \\
\hline & & & \multicolumn{2}{|c|}{ Firmeza de polpa (N) } & & \\
\hline 0 & $46,6 \mathrm{~b}$ & $71,6 \mathrm{aA}$ & $50,7 \mathrm{~b}$ & $73,3 \mathrm{aA}$ & $50,6 \mathrm{~b}$ & 80,7 aA \\
\hline 4 & $49,8 \mathrm{~b}$ & $71,6 \mathrm{aA}$ & $52,5 \mathrm{~b}$ & $72,6 \mathrm{aA}$ & $52,2 \mathrm{~b}$ & $78,7 \mathrm{aA}$ \\
\hline 8 & $50,0 \mathrm{~b}$ & $70,7 \mathrm{aA}$ & $53,4 \mathrm{~b}$ & $74,6 \mathrm{aA}$ & $48,8 \mathrm{~b}$ & $77,3 \mathrm{aA}$ \\
\hline 12 & $49,5 \mathrm{~b}$ & $70,5 \mathrm{aA}$ & $56,6 \mathrm{~b}$ & $72,9 \mathrm{aA}$ & $54,9 \mathrm{~b}$ & $76,1 \mathrm{aA}$ \\
\hline 16 & $49,8 \mathrm{~b}$ & $70,9 \mathrm{aA}$ & $50,5 \mathrm{~b}$ & $73,0 \mathrm{aA}$ & $50,1 \mathrm{~b}$ & $78,3 \mathrm{aA}$ \\
\hline 20 & $49,1 \mathrm{~b}$ & $69,3 \mathrm{aA}$ & $54,6 \mathrm{~b}$ & $72,0 \mathrm{aA}$ & $50,8 \mathrm{~b}$ & $77,1 \mathrm{aA}$ \\
\hline Média & $49,1 \mathrm{~b}$ & $70,8 \mathrm{a}$ & $53,1 \mathrm{~b}$ & $73,1 \mathrm{a}$ & $51,5 \mathrm{~b}$ & $77,8 \mathrm{a}$ \\
\hline \multirow[t]{2}{*}{ CV (\%) } & 4,7 & 1,3 & 5,4 & 1,9 & 4,1 & 3,0 \\
\hline & \multicolumn{6}{|c|}{ Acidez titulável (\% de ácido málico) } \\
\hline 0 & $0,083 \mathrm{~b}$ & $0,154 \mathrm{aAB}$ & $0,122 \mathrm{~b}$ & $0,172 \mathrm{aA}$ & $0,116 b$ & $0,190 \mathrm{aA}$ \\
\hline 4 & $0,091 \mathrm{~b}$ & $0,169 \mathrm{aA}$ & $0,096 \mathrm{~b}$ & $0,161 \mathrm{aA}$ & $0,105 \mathrm{~b}$ & $0,170 \mathrm{aA}$ \\
\hline 8 & $0,086 \mathrm{~b}$ & $0,169 \mathrm{aA}$ & $0,096 \mathrm{~b}$ & $0,171 \mathrm{aA}$ & $0,132 \mathrm{a}$ & $0,180 \mathrm{aA}$ \\
\hline 12 & $0,079 \mathrm{~b}$ & $0,164 \mathrm{aAB}$ & $0,103 \mathrm{~b}$ & $0,169 \mathrm{aA}$ & $0,107 \mathrm{a}$ & $0,167 \mathrm{aA}$ \\
\hline 16 & $0,086 \mathrm{~b}$ & $0,146 \mathrm{aBC}$ & $0,108 \mathrm{~b}$ & $0,171 \mathrm{aA}$ & $0,112 b$ & $0,170 \mathrm{aA}$ \\
\hline 20 & $0,088 \mathrm{a}$ & $0,133 \mathrm{aC}$ & $0,107 \mathrm{~b}$ & $0,154 \mathrm{aA}$ & $0,128 \mathrm{~b}$ & $0,170 \mathrm{aA}$ \\
\hline Média & $0,086 \mathrm{~b}$ & $0,156 \mathrm{a}$ & $0,106 \mathrm{~b}$ & $0,166 \mathrm{a}$ & $0,117 \mathrm{~b}$ & 0,174 \\
\hline \multirow[t]{2}{*}{ CV (\%) } & 4,4 & 6,6 & 11,3 & 8,3 & 11,6 & 10,0 \\
\hline & \multicolumn{6}{|c|}{ Sólidos solúveis (\%) } \\
\hline 0 & $12,8 \mathrm{~b}$ & $13,5 \mathrm{aA}$ & $14,6 \mathrm{a}$ & $14,7 \mathrm{aA}$ & $13,2 \mathrm{a}$ & $13,5 \mathrm{aA}$ \\
\hline 4 & $12,8 \mathrm{a}$ & $13,5 \mathrm{aA}$ & $14,0 \mathrm{~b}$ & $14,5 \mathrm{aA}$ & $13,0 \mathrm{a}$ & $13,5 \mathrm{aA}$ \\
\hline 8 & $12,8 \mathrm{a}$ & $13,4 \mathrm{aA}$ & $14,2 \mathrm{a}$ & $14,4 \mathrm{aA}$ & $13,6 \mathrm{a}$ & $13,3 \mathrm{aA}$ \\
\hline 12 & $12,6 \mathrm{a}$ & $13,4 \mathrm{aA}$ & $14,4 \mathrm{a}$ & $14,5 \mathrm{aA}$ & $13,1 \mathrm{a}$ & $13,5 \mathrm{aA}$ \\
\hline 16 & $12,9 \mathrm{~b}$ & $13,5 \mathrm{aA}$ & $14,3 \mathrm{a}$ & $14,5 \mathrm{aA}$ & $13,0 \mathrm{a}$ & $13,4 \mathrm{aA}$ \\
\hline 20 & $13,0 \mathrm{~b}$ & $13,5 \mathrm{aA}$ & $14,1 \mathrm{a}$ & $14,4 \mathrm{aA}$ & $13,4 \mathrm{a}$ & $13,7 \mathrm{aA}$ \\
\hline Média & $12,8 \mathrm{~b}$ & $13,5 \mathrm{a}$ & $14,3 \mathrm{a}$ & $14,5 \mathrm{a}$ & $13,2 \mathrm{~b}$ & 13,5 \\
\hline CV $(\%)$ & 1,5 & 1,0 & 3,4 & 2,3 & 2,4 & 2,1 \\
\hline
\end{tabular}

Médias seguidas de mesma letra, minúsculas nas linhas, para uma mesma região, e maiúscula nas colunas, nos tratamentos com 1-MCP, não diferem entre si, pelo teste LSD ( $\mathrm{p}<0,05)$. 
TABELA 3 - Incidência de escaldadura, escurecimento de polpa e podridões em maçãs 'Fuji Suprema', colhidas em diferentes regiões de produção e tratadas com 1-MCP após diferentes períodos de armazenamento refrigerado $\left(0,5 \pm 0,5^{\circ} \mathrm{C} / 90-95 \% \mathrm{UR}\right)$, sob atmosfera do ar, comparativamente ao tratamento-controle. Os frutos foram avaliados após nove meses de armazenamento refrigerado, mais sete dias a $23{ }^{\circ} \mathrm{C}$.

\begin{tabular}{|c|c|c|c|c|c|c|}
\hline \multirow{2}{*}{$\begin{array}{l}\text { Aplicação do 1-MCP } \\
\text { (dias após a colheita) }\end{array}$} & \multicolumn{2}{|c|}{ Bom Retiro } & \multicolumn{2}{|c|}{ Fraiburgo } & \multicolumn{2}{|c|}{ São Joaquim } \\
\hline & Controle & 1-MCP & Controle & 1-MCP & Controle & 1-MCP \\
\hline & \multicolumn{6}{|c|}{ Escaldadura superficial (\%) } \\
\hline 0 & $36,3 \mathrm{a}$ & $0,0 \mathrm{bA}$ & $82,0 \mathrm{a}$ & $0,0 \mathrm{bC}$ & $65,0 \mathrm{a}$ & $0,0 \mathrm{bB}$ \\
\hline 4 & $32,0 \mathrm{a}$ & $0,0 \mathrm{bA}$ & $81,4 \mathrm{a}$ & $0,0 \mathrm{bC}$ & $71,8 \mathrm{a}$ & $0,3 \mathrm{bB}$ \\
\hline 8 & $29,0 \mathrm{a}$ & $0,0 \mathrm{bA}$ & $82,3 \mathrm{a}$ & $0,7 \mathrm{bC}$ & $77,0 \mathrm{a}$ & $0,0 \mathrm{bB}$ \\
\hline 12 & 19,3 a & $0,0 \mathrm{bA}$ & $77,4 \mathrm{a}$ & $5,5 \mathrm{bC}$ & $66,3 \mathrm{a}$ & $1,5 \mathrm{bB}$ \\
\hline 16 & $22,7 \mathrm{a}$ & $0,0 \mathrm{bA}$ & $90,8 \mathrm{a}$ & $21,1 \mathrm{bB}$ & $82,0 \mathrm{a}$ & $17,7 \mathrm{bA}$ \\
\hline 20 & $21,0 \mathrm{a}$ & $0,0 \mathrm{bA}$ & $80,1 \mathrm{a}$ & $33,3 \mathrm{bA}$ & $85,1 \mathrm{a}$ & $23,4 \mathrm{bA}$ \\
\hline Média & $26,7 \mathrm{a}$ & $0,0 \mathrm{~b}$ & $82,4 \mathrm{a}$ & $10,1 \mathrm{~b}$ & $74,5 \mathrm{a}$ & $7,2 \mathrm{~b}$ \\
\hline \multirow[t]{2}{*}{ CV $(\%)$} & 24,7 & 0,0 & 8,2 & 34,5 & 12,2 & 58,0 \\
\hline & \multicolumn{6}{|c|}{ Escurecimento de polpa (\%) } \\
\hline 0 & $12,7 \mathrm{a}$ & $0,0 \mathrm{bA}$ & $12,1 \mathrm{a}$ & $1,0 \mathrm{bA}$ & $39,5 \mathrm{a}$ & $13,8 \mathrm{bAB}$ \\
\hline 4 & $8,3 \mathrm{a}$ & $0,0 \mathrm{bA}$ & $22,3 \mathrm{a}$ & $7,2 \mathrm{bA}$ & $56,8 \mathrm{a}$ & $16,7 \mathrm{bA}$ \\
\hline 8 & $5,3 \mathrm{a}$ & $0,0 \mathrm{bA}$ & $19,8 \mathrm{a}$ & $0,7 \mathrm{bA}$ & $31,1 \mathrm{a}$ & $2,7 \mathrm{bC}$ \\
\hline 12 & $12,3 \mathrm{a}$ & $0,0 \mathrm{bA}$ & $21,9 \mathrm{a}$ & $3,0 \mathrm{bA}$ & $50,3 \mathrm{a}$ & $2,9 \mathrm{bBC}$ \\
\hline 16 & $8,3 \mathrm{a}$ & $2,7 \mathrm{aA}$ & $27,8 \mathrm{a}$ & $1,0 \mathrm{bA}$ & $39,7 \mathrm{a}$ & $2,7 \mathrm{bC}$ \\
\hline 20 & $9,0 \mathrm{a}$ & $1,3 \mathrm{bA}$ & $25,1 \mathrm{a}$ & $2,8 \mathrm{bA}$ & $49,2 \mathrm{a}$ & $10,3 \mathrm{bABC}$ \\
\hline Média & $9,3 \mathrm{a}$ & $0,7 \mathrm{~b}$ & $21,5 \mathrm{a}$ & $2,6 \mathrm{~b}$ & $44,4 \mathrm{a}$ & $8,2 \mathrm{~b}$ \\
\hline \multirow[t]{2}{*}{ CV $(\%)$} & 33,8 & 61,6 & 26,4 & 49,0 & 20,5 & 43,5 \\
\hline & \multicolumn{6}{|c|}{ Podridões (\%) } \\
\hline 0 & $40,7 \mathrm{a}$ & $10,0 \mathrm{bA}$ & 48,9 a & $3,0 \mathrm{bA}$ & $25,7 \mathrm{a}$ & $1,3 \mathrm{bA}$ \\
\hline 4 & $37,7 \mathrm{a}$ & $13,0 \mathrm{bA}$ & $34,6 \mathrm{a}$ & $4,7 \mathrm{bA}$ & $28,6 \mathrm{a}$ & $0,0 \mathrm{bA}$ \\
\hline 8 & $26,3 \mathrm{a}$ & $7,7 \mathrm{bA}$ & $36,8 \mathrm{a}$ & $5,0 \mathrm{bA}$ & $38,5 \mathrm{a}$ & $1,4 \mathrm{bA}$ \\
\hline 12 & $37,0 \mathrm{a}$ & $4,4 \mathrm{bA}$ & $26,7 \mathrm{a}$ & $6,3 \mathrm{bA}$ & $19,0 \mathrm{a}$ & $5,8 \mathrm{bA}$ \\
\hline 16 & $20,3 \mathrm{a}$ & $9,3 \mathrm{aA}$ & $49,7 \mathrm{a}$ & $7,7 \mathrm{bA}$ & $20,5 \mathrm{a}$ & $5,4 \mathrm{bA}$ \\
\hline 20 & $24,7 \mathrm{a}$ & $5,0 \mathrm{bA}$ & $39,5 \mathrm{a}$ & $5,3 \mathrm{bA}$ & $26,1 \mathrm{a}$ & $4,4 \mathrm{bA}$ \\
\hline Média & $31,1 \mathrm{a}$ & $8,2 \mathrm{~b}$ & $39,4 \mathrm{a}$ & $5,3 \mathrm{~b}$ & $26,4 \mathrm{a}$ & $3,1 \mathrm{~b}$ \\
\hline CV $(\%)$ & 21,2 & 53,0 & 20,4 & 55,1 & 12,4 & 68,7 \\
\hline
\end{tabular}

Médias seguidas de mesma letra, minúsculas nas linhas, para uma mesma região, e maiúscula nas colunas, nos tratamentos com 1-MCP não diferem entre si, pelo teste $\operatorname{LSD}(\mathrm{p}<0,05)$. 


\section{CONCLUSÕES}

1-A eficiência do 1-MCP para prevenção de escaldadura superficial é reduzida quando sua aplicação for retardada por 16 ou 20 dias após a colheita, em maçãs 'Fuji Suprema' colhidas nas regiões de Fraiburgo e São Joaquim e armazenadas sob atmosfera do ar por nove meses, a $0,5^{\circ} \mathrm{C}$.

2-A eficiência do 1-MCP para conservação da firmeza da polpa e prevenção do escurecimento da polpa e podridões em maçãs 'Fuji Suprema' não é reduzida quando sua aplicação for retardada por até 20 dias após a colheita, independentemente da região de produção.

3-Os efeitos do retardo na aplicação do 1-MCP sobre sua eficiência na conservação da acidez e teor de sólidos solúveis em maçãs 'Fuji Suprema' dependem da região de produção dos frutos.

\section{AGRADECIMENTOS}

À AgroFresh Inc., pelo apoio e fornecimento do 1-MCP (SmartFresh $\left.{ }^{\mathrm{SM}}\right)$.

\section{REFERÊNCIAS}

ABPM. Informações estatísticas. Disponível em: $<$ http://www.abpm.org.br/informações.htm.> Acesso em: 10 ago. 2009.

AKBUDAK, B.; OZER, M.H.; ERTURK, U.; CAVUSOGLU, S. Response of 1-methylcyclopropene treated "Granny Smith" apple fruit to air and controlled atmosphere storage conditions. Journal of Food Quality, Boston, v.32, n.1, p.18-33, 2009.

ARGENTA, L.C.; BRAKMANN, A.; MONDARDO, M. Qualidade pós-colheita de maçãs armazenadas sob diferentes temperaturas e concentrações de $\mathrm{CO}_{2}$ e $\mathrm{O}_{2}$. Revista Brasileira de Fisiologia Vegetal, Brasília, v.6, n.2, p.121-126, 1994.

ARGENTA, L.C.; FAN, X.; MATTHEIS, J. Factors affecting efficacy of 1-MCP to maintain quality of apples fruit after storage. Acta Horticulturae, Leuven, n.682, p.1249-1255, 2005.
ARGENTA, L.C.; FAN, X.; MATTHEIS, J. Responses of 'Fuji' apples to short and long duration exposure to high $\mathrm{CO}_{2}$. Postharvest Biology and Technology, Amsterdam, v.24, n.1, p.13-24, 2002.

ARGENTA, L.C.; FAN, X.; MATTHEIS, J.P. Development of internal browning in 'Fuji' apples during storage. In: WASHINGTON TREE FRUIT POSTHARVEST CONFERENCE, 2001, Wenatchee, EUA. Proceedings.... Disponível em: $<$ http:// postharvest.tfrec.wsu.edu/PC2001C.pdf > . Acesso em :29 set. 2001b.

ARGENTA, L.C.; MATTHEIS, J.; FAN, X. Retardamento da maturação de maçãs 'Fuji' pelo tratamento com 1-MCP e manejo da temperatura. Revista Brasileira de Fruticultura, Jaboticabal, v.23, n.2, p.270-273, 2001a.

ARGENTA, L.C.; MONDARDO, M. Maturação na colheita e qualidade de maçãs Gala após a armazenagem. Revista Brasileira de Fisiologia Vegetal, Brasília, v.6, n.2, p.135-140, 1994.

BRASIL. Ministério Da Agricultura, Pecuária E Abastecimento. Regulamento técnico de identidade e qualidade da maçã. Brasília, 2006. 9p. (Instrução Normativa, 5).

DeELL, J.R.; MURR, D.P.; PORTEOUS, M.D.; RUPASINGHE, H.P.V. Influence of temperature and duration of 1-methylcyclopropene (1-MCP) treatment on apple quality. Postharvest Biology and Technology, Amsterdam, v.24, n.3, p.349-353, 2002.

EMONGOR, V.E.; MURR, D.P.; LOUGHEED, E.C. Preharvest factors that predispose apples to superficial scald. Postharvest Biology and Technology, Amsterdam, v.4, n.4, p.289-300, 1994.

FAN, X.; BLANKENSHIP, S.M.; MATTHEIS, J.P. 1-Methylcyclopropene inhibits apple ripening. Journal of American Society for Horticultural Science, Alexandria, v.124, n.6, p.690-695, 1999a.

FAN, X.; MATTHEIS, J.P.; BLANKENSHIP, S. Development of superficial scald, coreflush, and peel greasiness is reduced by MCP. Journal of Agricultural and Food Chemistry, Washington, v.47, n.8, p.3063-3068, 1999 b. 
JU, Z.; CURRY, E.A. Evidence that alpha-farnesene biosynthesis during fruit ripening is mediated by ethylene regulated gene expression in apples. Postharvest Biology and Technology, Amsterdam, v.19, n.1, p.9-16, 2000.

JUNG, S.-K.; WATKINS, C.B. Superficial scald control after delayed treatment of apple fruit with diphenylamine (DPA) and 1-methylcyclopropene (1-MCP). Postharvest Biology and Technology, Amsterdam, v.50, n.1, p.45-52, 2008.

PECHOUS, S.W.; WATKINS, C.B.; WHITAKER, B.D. Expression of $\alpha$-farnesene synthase gene AFS 1 in relation to levels of $\alpha$-farnesene and conjugated trienols in peel tissue of scald-susceptible 'Law Rome' and scald-resistant 'Idared' apple fruit. Postharvest Biology and Technology, Amsterdam, v.35, n.2, p.125-132, 2005.

RUPASINGHE, H.P.V.; MURR, D.P.; PALIYATH, G.; SKOG, L. Inhibitory effect of 1-MCP on ripening and superficial scald development in 'McIntosh' and 'Delicious' apples. Journal of Horticultural Science Biotechnology, Kent, v.75, n.3, p.271-276, 2000.

SAFTNER, R.A.; ABBOTT, J.A.; CONWAY, W.S.; BARDEN, C.L. Effects of 1-methylcyclopropene and heat treatments on ripening and postharvest decay in 'Golden Delicious' apples. Journal of the American Society for Horticultural Science, Alexandria, n.128, n.1, p.120-127, 2003.

SAS INSTITUTE. Getting started with the SAS learning edition. Cary, 2002. 200p.
TSANTILI, E.; GAPPER, N.E.; ARQUIZA, J.M.R.A.; WHITAKER, B.D.; WATKINS, C.B. Ethylene and $\alpha$-farnesene metabolism in green and red skin of three apple cultivars in response to 1-methylcyclopropene (1-MCP) treatment. Journal of Agricultural and Food Chemistry, Davis, v.55, n.13, p.5267-5276, 2007.

VA L DEB ENITO SANHUEZA, R.M Recomendações para o controle pós-colheita das podridões de maçãs. Bento Gonçalves: EMBRAPACNPUV, 1996. 4p. (Comunicado Técnico, 21)

VALDEBENITO SANHUEZA， R.M.; MAFFIOLETTI, M.; COMPARIM，C.C.; KRASNIAK, J.; BOGO, A.; ARCARI, R. Características e controle da podridão "olho de boi” nas maçãs do Sul do Brasil. Bento Gonçalves: EMBRAPA-CNPUV, 2006. 12p. (Circular Técnica, 66)

WATKINS, C.B.; NOCK, J.F. Effects of delays between harvest and 1-methylcyclopropene treatment, and temperature during treatment, on ripening of air-stored and controlled-atmospherestored apples. HortScience, Alexandria, v.40, n.7, p.2096-2101, 2005.

WATKINS, C.B.; NOCK, J.F.; WHITAKER, B.D. Responses of early, mid and late season apple cultivars to postharvest application of 1-methylcyclopropene (1-MCP) under air and controlled atmosphere storage conditions. Postharvest Biology and Technology, Amsterdam, v.19, n.1, p.17-32, 2000.

ZANELLA, A. Control of apple superficial scald and ripening - a comparison between 1-methylcyclopropene and diphenylamine postharvest treatments, initial low oxygen stress and ultra low oxygen storage. Postharvest Biology and Technology, Amsterdam, v.27, n.1, p.69-78, 2003. 